\title{
Caracterización del sotobosque en bosques densos de pino y oyamel en el Nevado de Toluca, México
}

\author{
Characterization of the understory in dense pine and fir forests of the \\ Nevado de Toluca
}

Ana Mejía Canales', Sergio Franco-Maass, Angel Rolando Endara Agramont' y Víctor Ávila Akerberg'

Universidad Autónoma del Estado de México.
Instituto de Ciencias Agropecuarias y Rurales.
Toluca, Estado de México, México.
* Autor de correspondencia. sfrancom@uaemex.mx

\section{RESUMEN}

La mayor diversidad florística de los bosques templados se encuentra en el componente herbáceo y arbustivo del sotobosque, en estos, la flora adquiere gran importancia ecológica y funcional. Los procesos esenciales del ciclo de nutrientes y la trayectoria sucesional, dependen directamente de la vegetación del sotobosque. Por ello, es necesario realizar la caracterización florística de estas comunidades y conocer su diversidad. En este estudio se determinó la composición y estructura de las especies herbáceas y arbustivas en bosques densos de Pinus hartwegii (Lindl) y Abies religiosa (Kunth Schltdl. et Cham.) del Nevado de Toluca y se cuantificó la abundancia de regeneración arbórea en 10 cuadrantes continuos de $10 \mathrm{~m} \times 10 \mathrm{~m}$. En las especies menores a $50 \mathrm{~cm}$ de altura se estimó la cobertura y abundancia con base en la escala Braun-Blanquet. En las especies mayores a $50 \mathrm{~cm}$ se obtuvo el valor relativo en cobertura, frecuencia y densidad, además del valor de importancia. De acuerdo con los resultados obtenidos, la flora del sotobosque de Abies religiosa posee una mayor riqueza y está compuesta por 33 especies, 25 géneros y 14 familias, donde Asteraceae es la familia dominante. El sotobosque de Pinus hartwegii registró un total de 18 especies, 16 géneros y 10 familias, con dominancia en cobertura y abundancia de la familia Poaceae. En ambas comunidades vegetales la abundancia de la regeneración es mayor en plántulas y menor en brinzales y latizales.

PALABRAS CLAVE: Abies religiosa, composición florística, estructura del sotobosque, Pinus hartwegii, regeneración forestal, sotobosque.

\section{ABSTRACT}

The greatest floristic diversity of the temperate forests is found in the herbaceous and shrubby component of the undergrowth, in which the flora acquires great ecological and functional importance. Essential processes of the nutrient cycle and the successional trajectory, depend directly on the vegetation of the understory. This study determined the composition and structure of the herbaceous and shrub species in dense forests of Pinus hartwegii (Lindl) and Abies religiosa (Kunth Schltdl and et Cham.) of the Nevado de Toluca and quantified the abundance of tree regeneration in 10 quadrants continuous of $10 \mathrm{~m} \times 10 \mathrm{~m}$. In the species smaller than $50 \mathrm{~cm}$ the coverage and abundance was estimated based on the Braun-Blanquet scale and, in the species greater than $50 \mathrm{~cm}$, the relative value in coverage, frequency and density were obtained, besides their value of importance. According to our results, the understory of the forests of $A$ Abies religiosa is greater in diversity and is composed of 33 species, 25 genera and 14 families, where Asteraceae is the dominant family. The understory of Pinus hartwegii is composed by 18 species, 16 genera and 10 families, with dominance in coverage and abundance of the family Poaceae. In both, plant communities' regeneration is greater in seedlings and less in small and large saplings.

KEYWORDS: Abies religiosa, floristic composition, undergrowth structure, Pinus hartwegii, forest regeneration, understory. 


\section{INTRODUCCIÓN}

La vegetación que se desarrolla debajo del dosel arbóreo, comúnmente llamada sotobosque, tiene entre sus principales elementos estructurales a las briofitas, los líquenes, los helechos, las plántulas arbóreas, las herbáceas y los arbustos (Antos, 2017). En el bosque templado, el sotobosque cuenta con una importante diversidad de especies vegetales y juega un rol importante en la dinámica del ecosistema (Bartels y Chen, 2010).

El sotobosque es esencial para procesos del ciclo de nutrientes, la trayectoria sucesional o el régimen de fuego es el hábitat o fuente de alimento para numerosos vertebrados e invertebrados (Gilliam, 2007). En este, algunas especies crean condiciones de interferencia en las plántulas arbóreas que varían en competencia, alelopatía, inhibición micorrícica y obstrucción física (Royo y Carson, 2006).

El hábitat en el sotobosque es heterogéneo y dinámico, con un ambiente variable que depende de las características del dosel y de los recursos en el medio (Augusto, Dupouey y Ranger, 2003; Hart y Chen, 2006). En los bosques de coníferas, el sotobosque presenta regularmente una elevada humedad, con poca luz, viento y escasos nutrientes en el suelo. En dichas comunidades la tolerancia hacia los suelos ácidos es amplia (Candan, Broquen y Pellegrini, 2006; Rzedowski, 2006).

Con respecto a la composición florística, el sotobosque de las coníferas presenta una amplia riqueza de especies. En los bosques de Pinus hartwegii, los componentes dominantes son las gramíneas de géneros como Festuca, Calamagrostis y Mublenbergia. Eventualmente se encuentran especies del género Lupinus como resultado de la ocurrencia de incendios (Rzedowski, 2006). En el bosque de Abies religiosa, por el contrario, el sotobosque es poco abundante en gramíneas. En ambos bosques, los representantes de la familia Asteraceae son numerosos (Encina-Domínguez, Encina-Domínguez, Mata-Rocha y Valdes-Reyna, 2008).

En el área de protección de flora y fauna Nevado de Toluca (Apffnt), los bosques de pino y oyamel ocupan 66\% de la superficie forestal (Endara, 2010), donde 7815 ha son bosques densos de pino y 13040 ha bosques densos de oyamel (Franco, Regil, González y Nava, 2006). En la estructura espacial los bosques densos de Pinus hartwegii y Abies religiosa presentan tres estratos verticales que llegan a los $30 \mathrm{~m}$ y $48 \mathrm{~m}$ respectivamente. La densidad promedio de individuos en el bosque de pino es de $336 \mathrm{ha}^{-1} \mathrm{y}$ de 637 $\mathrm{ha}^{-1}(\geq 2.5 \mathrm{~cm}$ DAP) en el bosque de oyamel (Endara, Franco, Nava, Valdez y Fredericksen, 2012).

\section{OBJETIVOS}

En el Nevado de Toluca existen extensas regiones dominadas por especies arbóreas nativas, que definen la fisonomía de la comunidad (Mastretta et al., 2014), por ello es necesario caracterizar su composición vegetal y promover la conservación de sus especies. En el presente trabajo se planteó, como objetivo central, determinar la composición y estructura de herbáceas y arbustos en áreas de bosque denso de Pinus hartwegii y Abies religiosa del Nevado de Toluca. Adicionalmente se cuantificó la densidad de la regeneración arbórea.

\section{MATERIALES Y MÉTODOS}

El Apffnt se localiza en el Estado de México, hacia el suroeste del valle de Toluca y abarca parte de los municipios de Almoloya de Juárez, Amanalco de Becerra, Calimaya, Coatepec Harinas, Temascaltepec, Tenango del Valle, Toluca, Villa Guerrero, Villa Victoria y Zinacantepec (Gobierno del Estado de México [GEM], 1999). Forma parte del sistema volcánico transmexicano y alcanza una altitud de $4660 \mathrm{~m}$ snm. En el área predomina el tipo de suelo Andosol y en menor proporción Feozem, Regosol, Cambisol y Litosol. De los $3700 \mathrm{~m}$ snm en adelante predomina el clima frío y, entre los $2800 \mathrm{~m}$ y los $3700 \mathrm{~m}$ snm, el clima semifrío subhúmedo con lluvias en verano. En el Apffnt la riqueza de coníferas comprende especies de pino: Pinus hartwegii Lindl., P. montezumae Lamb., P. pseudostrobus Lindl., entre otros, así como oyamel (Abies religiosa Kunth Schltdl. \& Cham.), Juniperus flaccida Schlechtendal y Cupressus lusitánica Mill. Además, se desarrollan bosques de Quercus laurina Humb et Bonpl. y Alnus jorullensis Humboldt, Bonpland \& Kunth, así como 
zonas de pastizal, zacatonal alpino o páramo de altura (Comisión Nacional de Áreas Naturales Protegidas, 2013). El muestreo de la vegetación se realizó en los meses de agosto a octubre de 2015, en las localidades de Agua Blanca y Santa María del Monte (Zinacantepec) (Fig. 1). Las zonas de bosque denso de Abies religiosa y Pinus hartwegii se diferenciaron previamente con la ayuda de imágenes de satélite multiespectrales. En el área, se considera bosque denso a aquella superficie que cubre entre $80 \%$ y $100 \%$ de cobertura de copa, identificado sobre un cuadrante cartografiable de $100 \mathrm{~m}^{2}$ (Regil, Franco, Nava y Ordóñez, 2015).

En cada sitio de muestreo se establecieron 10 cuadrantes de $10 \mathrm{~m} \times 10 \mathrm{~m}\left(1000 \mathrm{~m}^{2}\right)$ en un gradiente continuo y en cada uno se registraron altitud, pendiente, coordenadas geográficas y tipo de perturbación asociada. Para confirmar la representatividad del muestreo, se realizó una curva de acumulación de especies en cada comunidad vegetal (Fig. 2) y, para analizar la composición, se realizó un método discriminativo por altura; en las especies mayores a $50 \mathrm{~cm}$, se obtuvieron los valores relativos en cobertura, densidad, frecuencia y valor de importancia (Mostacedo y Fredericksen, 2000). De las especies menores a $50 \mathrm{~cm}$ se estimó la cobertura y abundancia con base en la escala Braun-Blanquet (Braun-Blanquet, 1979).

Por la elevada densidad de gramíneas (mayores a 50 $\mathrm{cm}$ ) en el bosque de pino, el muestreo se estandarizó al método Braun-Blanquet. Finalmente, para evaluar la densidad de regeneración arbórea, se cuantificó el número de plántulas $(<30 \mathrm{~cm}$ de altura), brinzales $(\geq 30 \mathrm{~cm}<1.5$ $\mathrm{m}$ altura) y latizales $(\geq 1.5 \mathrm{~m}<2.5 \mathrm{~cm}$ de DAP) (Valdez, 2002).

De cada especie vegetal registrada, se recolectaron ejemplares por triplicado para su posterior herborización y determinación taxonómica con claves especializadas. En el Herbario-Hortorio (Chapa) del Campus Montecillo del Colegio de Postgraduados, se depositaron duplicados de los ejemplares recolectados y se confirmó la determinación de especímenes. La agrupación de familias se realizó de acuerdo con el sistema Angiosperm Phylogeny Group [APG IV]
(2016) y para las abreviaturas de los autores de las especies se utilizó el Índice Internacional de Nombres de Plantas (The International Plant Names Index [IPNI], 2017). El nombre común de las especies, se obtuvo por consulta a las personas de las localidades adyacentes a las zonas de muestreo y se complementó con bibliografía especializada (Rzedowski, Rzedowski y colaboradores, 2005; CornejoTenorio e Ibarra-Manríquez, 2008). La agrupación de especies en gremios ecológicos se realizó de forma preliminar con base en las características ecológicas y foliares de la especie (Givnish, 1988; Grime, 1989).

\section{Resultados}

\section{Composición florística}

En el bosque de Abies religiosa, la flora del sotobosque cuenta con un total de 33 especies, 25 géneros y 14 familias agrupadas en 24 herbáceas, ocho arbustos y un árbol (Tabla 1).

El bosque de Pinus hartwegii se caracteriza por un sotobosque con 18 especies, 16 géneros y nueve familias, que se agrupan en 15 herbáceas, dos arbustos y un árbol (Tabla 2).

En cuanto a la regeneración arbórea de las áreas evaluadas se encontró que esta es exclusiva de las especies de oyamel y pino, las plántulas fueron dominantes en densidad, con $87.2 \%$ en Abies y $47 \%$ en Pinus, seguidas por brinzales $(12.2 \%$ Abies y $41.2 \%$ Pinus) y latizales $(0.6 \%$ Abies y $11.8 \%$ Pinus).

\section{Estructura del sotobosque}

En el sotobosque de Abies religiosa, las herbáceas: Stevia monardifolia, Salvia gracilis y Sigesbeckia jorullensis son dominantes en la altura mayor a $50 \mathrm{~cm}$, en este nivel también predominan los arbustos Roldana angulifolia y Cestrum anagyris. En las herbáceas menores a $50 \mathrm{~cm}$, Stevia monardifolia, Roldana platanifolia y Sibthorpia repens tienen la mayor cobertura y abundancia, mientras que los arbustos Roldana angulifolia, Acaena elongata y Fuchsia microphylla son dominantes (Tabla 3 y Fig. 3). 


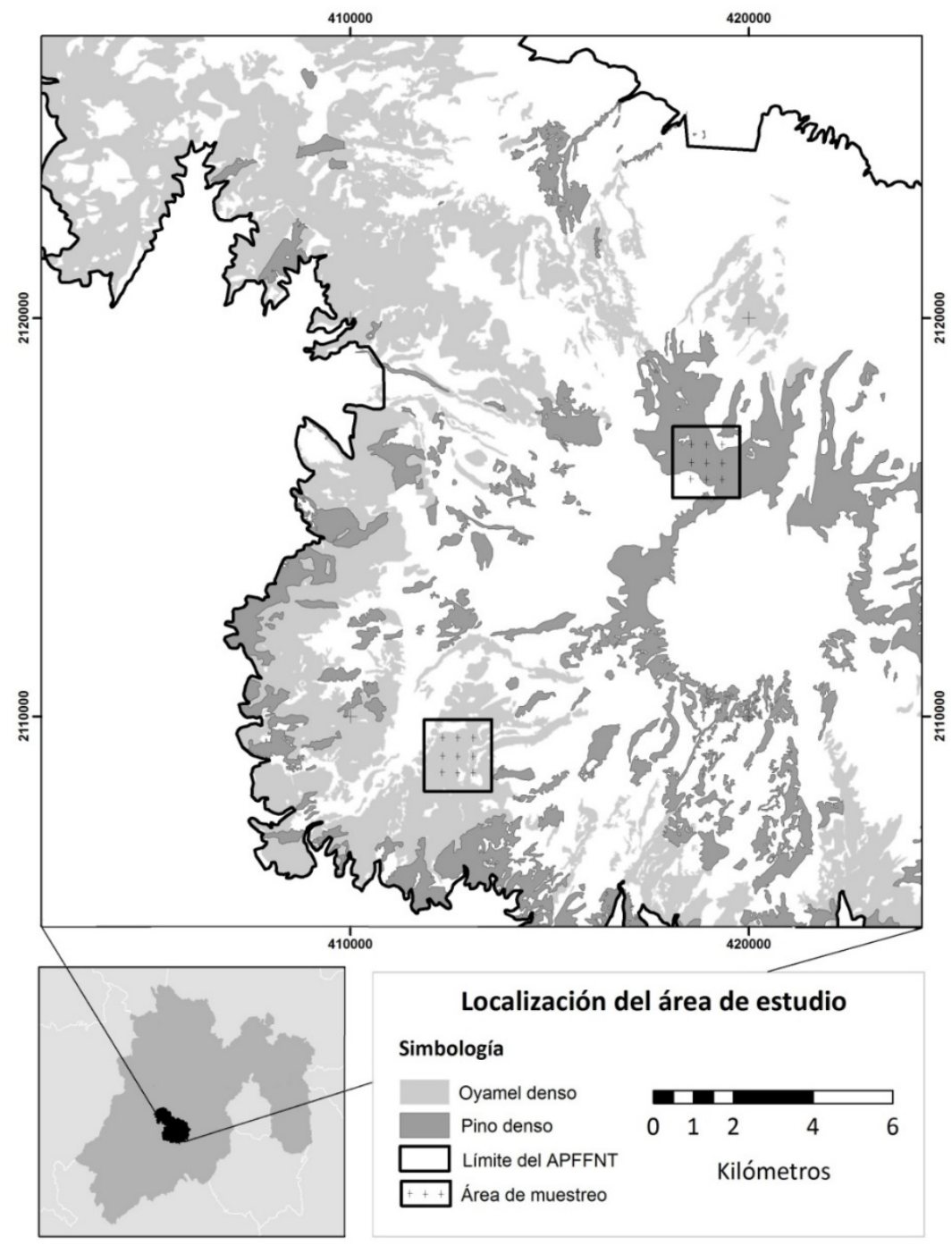

FIGURA 1. Ubicación de los sitios de muestreo

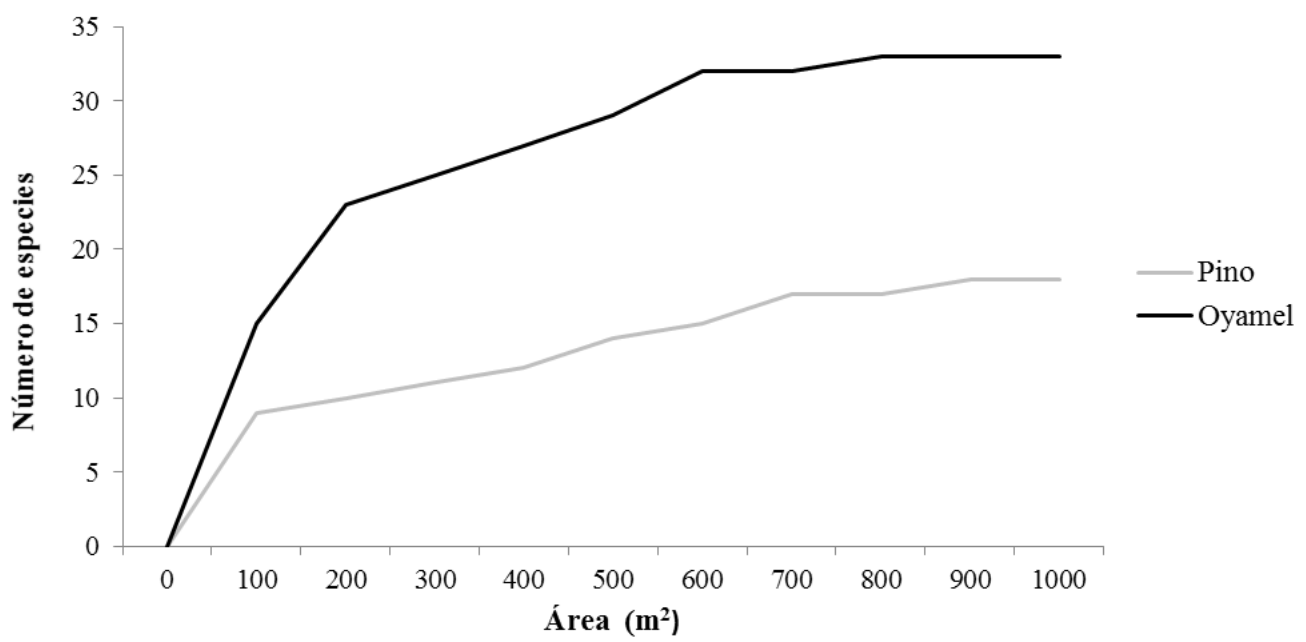

FIGURA 2. Curva de acumulación de especies para el sotobosque de bosques densos de pino y oyamel. 
TABLA 1. Flora vascular del sotobosque en el bosque denso de Abies religiosa del Nevado de Toluca.

\begin{tabular}{|c|c|c|c|}
\hline FAMILIA/Nombre científico & Nombre común & Forma de vida & Gremio ecológico \\
\hline $\begin{array}{l}\text { APIACEAE } \\
\text { Osmorhiza aff. mexicana Griseb. } \\
\text { ASTERACEAE }\end{array}$ & S/D & Herbácea & Heliófita \\
\hline Ageratina aff. parayana ${ }^{2}$ (Espinosa) B. L. Turner & Raíz de serpiente & Herbácea & Esciófita \\
\hline Ageratina pazcuarensis (Kunth) R. M. King \& H. Rob. & Hierba del ángel & Herbácea & Esciófita \\
\hline Ageratina rivalis (Greenm.) R. M. King \& H. Rob. & $S / D$ & Arbusto & Heliófita \\
\hline Bidens triplinervia' Kunth & Acahual cimarrón & Herbácea & Heliófita \\
\hline Cirsium ehrenbergii'12 Sch. Bip. & Cardo santo & Herbácea & Esciófita \\
\hline Piqueria pilosa ${ }^{2}$ Kunth & Cardón pelón & Herbácea & Esciófita \\
\hline Roldana angulifolia (DC.) H. Rob. \& Brettell & Hoja ancha & Arbusto & Esciófita \\
\hline Roldana lineolata ${ }^{12}$ (DC.) H. Rob. \& Brettell & S/D & Herbácea & Esciófita \\
\hline Roldana platanifolia ${ }^{2}$ (Benth.) H. Rob. \& Brettell & $\begin{array}{l}\text { Hierba del zopilote, mano de } \\
\text { león }\end{array}$ & Herbácea & Esciófita \\
\hline Roldana reticulata ${ }^{2}$ (DC.) H. Rob. \& Brettell & S/D & Herbácea & Esciófita \\
\hline Senecio callosus Sch. Bip. & Hoja de flecha & Herbácea & Esciófita \\
\hline Senecio cinerarioides ${ }^{12}$ Kunth & Jara, Jarilla blanca & Arbusto & Heliófita \\
\hline Sigesbeckia jorullensis' Kunth & Flor de araña & Herbácea & Esciófita \\
\hline Stevia monardifolia ${ }^{2}$ Kunth & S/D & Herbácea & Esciófita \\
\hline \multicolumn{4}{|l|}{ CAPRIFOLIACEAE } \\
\hline Symphoricarpos microphyllus Kunth & Perlilla, Perlitas, Escobilla & Arbusto & Heliófita \\
\hline \multicolumn{4}{|l|}{ CARYOPHYLLACEAE } \\
\hline Cerastium nutans Raf. & S/D & Herbácea & Esciófita \\
\hline Stellaria cuspidata Willd. & S/D & Herbácea & Esciófita \\
\hline \multicolumn{4}{|l|}{ DRYOPTERIDACEAE } \\
\hline $\begin{array}{l}\text { Plecosorus speciosissimus (A. Braun ex Kunze) T. } \\
\text { Moore }\end{array}$ & S/D & Herbácea & Esciófita \\
\hline \multicolumn{4}{|l|}{ GERANIACEAE } \\
\hline Geranium seemannii Peyr. & Mano de león & Herbácea & Esciófita \\
\hline \multicolumn{4}{|l|}{ LAMIACEAE } \\
\hline Salvia gracilis Benth. & S/D & Herbácea & Esciófita \\
\hline Salvia lavanduloides Kunth & Tepechia & Herbácea & Esciófita \\
\hline Salvia aff. microphylla Kunth & Bandera mexicana & Arbusto & Esciófita \\
\hline \multicolumn{4}{|l|}{ ONAGRACEAE } \\
\hline Fuchsia microphylla Kunth & Aretillo & Arbusto & Esciófita \\
\hline \multicolumn{4}{|l|}{ PINACEAE } \\
\hline $\begin{array}{l}\text { Abies religiosa (Kunth) Schltdl. \& Cham. } \\
\text { POACEAE }\end{array}$ & Oyamel, abeto & Árbol & Esciófita \\
\hline Deschampsia sp. & S/D & Herbácea & Esciófita \\
\hline Trisetum irazuense Hitchc. & S/D & Herbácea & Esciófita \\
\hline \multicolumn{4}{|l|}{ ROSACEAE } \\
\hline Acaena elongata' L. & Pegarropa, cadillo & Arbusto & Esciófita \\
\hline Alchemilla procumbens' Rose & Chinilla, pata de león & Herbácea & Esciófita \\
\hline \multicolumn{4}{|l|}{ RUBIACEAE } \\
\hline Didymaea alsinoides (Schltdl. \& Cham.) Standl. & Ocoxóchitl & Herbácea & Esciófita \\
\hline Galium aschenbornii S. Schauer & Cuajaleche, pegarropa & Herbácea & Esciófita \\
\hline \multicolumn{4}{|l|}{ SCROPHULARIACEAE } \\
\hline Sibthorpia repens (L.) Kuntze & Pepita & Herbácea & Esciófita \\
\hline \multicolumn{4}{|l|}{ SOLANACEAE } \\
\hline Cestrum anagyris Dunal & Hierba del zopilote & Arbusto & Esciófita \\
\hline
\end{tabular}


TABLA 2. Flora vascular del sotobosque en el bosque denso de Pinus hartwegii del Nevado de Toluca.

\begin{tabular}{|c|c|c|c|}
\hline FAMILIA/ Nombre científico & Nombre común & Forma de vida & Gremio ecológico \\
\hline \multicolumn{4}{|l|}{ ASTERACEAE } \\
\hline Cirsium jorullense² Spreng. & Cardo santo & Herbácea & Esciófita \\
\hline $\begin{array}{l}\text { Pseudognaphalium liebmannii (Sch. Bip. } \\
\text { ex Klatt) }\end{array}$ & $\begin{array}{l}\text { Gordolobo, hierbabuena, } \\
\text { altamisa }\end{array}$ & Herbácea & Esciófita \\
\hline Senecio cinerarioides ${ }^{12}$ Kunth & Jara, Jarilla blanca & Arbusto & Heliófita \\
\hline Senecio iodanthus ${ }^{2}$ Greenm. & $S / D$ & Herbácea & Esciófita \\
\hline Senecio roseus ${ }^{2}$ Sch. Bip. & Sierrilla & Herbácea & Esciófita \\
\hline Stevia incognita Grashoff & Yoloquilde & Herbácea & Esciófita \\
\hline \multicolumn{4}{|l|}{ CARYOPHYLLACEAE } \\
\hline Arenaria bryoides D. F. K. Schltdl. & $S / D$ & Herbácea & Esciófita \\
\hline \multicolumn{4}{|l|}{ ERICACEAE } \\
\hline Vaccinium caespitosum Michx. & Arándanos, Borrachos & Arbusto & Esciófita \\
\hline \multicolumn{4}{|l|}{ FABACEAE } \\
\hline Lupinus montanus' Kunth & Garbancillo, matapiojo & Herbácea & Heliófita \\
\hline \multicolumn{4}{|l|}{ OXALIDACEAE } \\
\hline Oxalis alpina Rose & Xocoyotl & Herbácea & Esciófita \\
\hline \multicolumn{4}{|l|}{ PINACEAE } \\
\hline Pinus hartwegii Lindl. & Ocote & Árbol & Heliófita \\
\hline \multicolumn{4}{|l|}{ POACEAE } \\
\hline Agrostis tolucensis Kunth & $S / D$ & Herbácea & Heliófita \\
\hline $\begin{array}{l}\text { Calamagrostis tolucensis (Kunth) Trin. ex } \\
\text { Steud. }\end{array}$ & Zacatón & Herbácea & Heliófita \\
\hline Cinna poiformis (Kunth) Scribn. \& Merr. & $S / D$ & Herbácea & Heliófita \\
\hline Festuca tolucensis Kunth & Zacatón & Herbácea & Heliófita \\
\hline Trisetum spicatum (L.) K. Richt. & S/D & Herbácea & Heliófita \\
\hline \multicolumn{4}{|l|}{ ROSACEAE } \\
\hline Alchemilla procumbens ${ }^{1}$ Rose & Chinilla, pata de león & Herbácea & Esciófita \\
\hline \multicolumn{4}{|l|}{ SCROPHULARIACEAE } \\
\hline Penstemon gentianoides Poir. & Jarritos, campanita morada & Herbácea & Esciófita \\
\hline
\end{tabular}

"1" especies asociadas a disturbio, "2" especies endémicas del país, "S/D" sin dato. 
TABLA 3. Especies dominantes del sotobosque en el bosque denso de Abies religiosa*.

\begin{tabular}{lcccc}
\hline Estrato $\geq 50 \mathrm{~cm}$ & & & \\
\hline Valor de importancia & & & & \\
& & & & \\
Herbáceas & & & & \\
Stevia monardifolia & 34.4 & 46.6 & 8.3 & 38.1 \\
Salvia gracilis & 39.1 & 20.9 & 25 & 22.8 \\
Sigesbeckia jorullensis & 17.2 & 17.7 & 20 \\
Arbustos & & & 40 & \\
Roldana angulifolia & 94.6 & 93.1 & 40 & 75.9 \\
Cestrum anagyris & 4.6 & 6.3 & & 17 \\
\hline Estrato $\leq 50 \mathrm{~cm}$ & & & & \\
\hline
\end{tabular}

Escala Braun-Blanquet

$\begin{array}{lllllllllll}\text { Cuadrante } & 1 & 2 & 3 & 4 & 5 & 6 & 7 & 8 & 9 & 10\end{array}$

Herbáceas

Stevia monardifolia

Roldana platanifolia

Sibthorpia repens

Ageratina pazcuarensis

Ageratina aff. parayana

Sigesbeckia jorullensis

Trisetum irazuense

Piqueria pilosa

Arbustos

Roldana angulifolia

Acaena elongata

Fuchsia microphylla

Cestrum anagyris

$\begin{array}{llllllllll}2 & 4 & 2 & 4 & 3 & 2 & 3 & 3 & 3 & 2 \\ 3 & 3 & 4 & 5 & 3 & 2 & 2 & 2 & 2 & + \\ 3 & 2 & 1 & 3 & + & 3 & 3 & 3 & 2 & 1 \\ - & + & - & 3 & 3 & 2 & 4 & 3 & 2 & 3 \\ + & - & - & 2 & + & + & 3 & + & 4 & 4 \\ + & - & - & 2 & + & 2 & + & 3 & 2 & + \\ + & 1 & + & + & + & 1 & + & + & + & + \\ + & - & - & - & 2 & + & 2 & 2 & 1 & -\end{array}$

* Se indica el valor de importancia o la estimación de cobertura y abundancia.

Escala Braun-Blanquet: cobertura de la especie; (-) ausencia, (+) pocos individuos con baja cobertura, (1) <5\%, (2) 5 - $25 \%$, (3) 25 - $50 \%$, (4) 50 - $75 \%$. Los resultados señalados en la tabla consideran a las especies dominantes, las especies restantes se omitieron por ser poco informativas.

Con respecto al sotobosque en el bosque denso de Pinus hartwegii estudiado, las herbáceas dominantes en la altura mayor a $50 \mathrm{~cm}$ son Calamagrostis tolucensis, Agrostis tolucensis, Penstemon gentianoides y Lupinus montanus, en esta categoría, la única especie arbustiva dominante es Senecio cinerarioides. En el nivel menor a $50 \mathrm{~cm}$ Alchemilla procumbens y Penstemon gentianoides son las herbáceas dominantes (Tabla 4 y Fig. 4). 


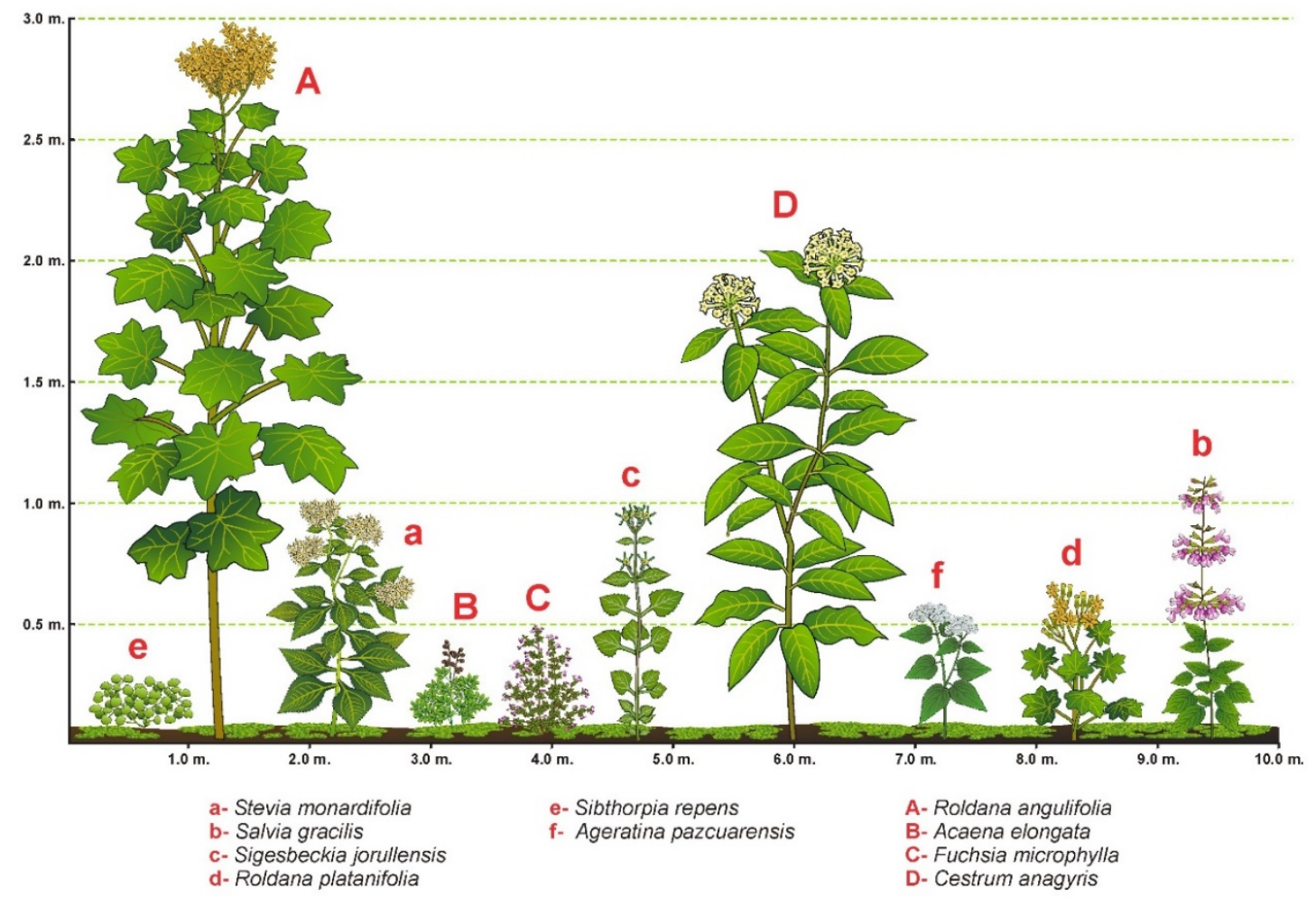

FIGURA 3. Estructura del sotobosque en el bosque de Abies religiosa, se presenta la altura y cobertura aproximada de las especies dominantes.

Tabla 4. Especies dominantes del sotobosque en el bosque denso de Pinus hartwegii*.

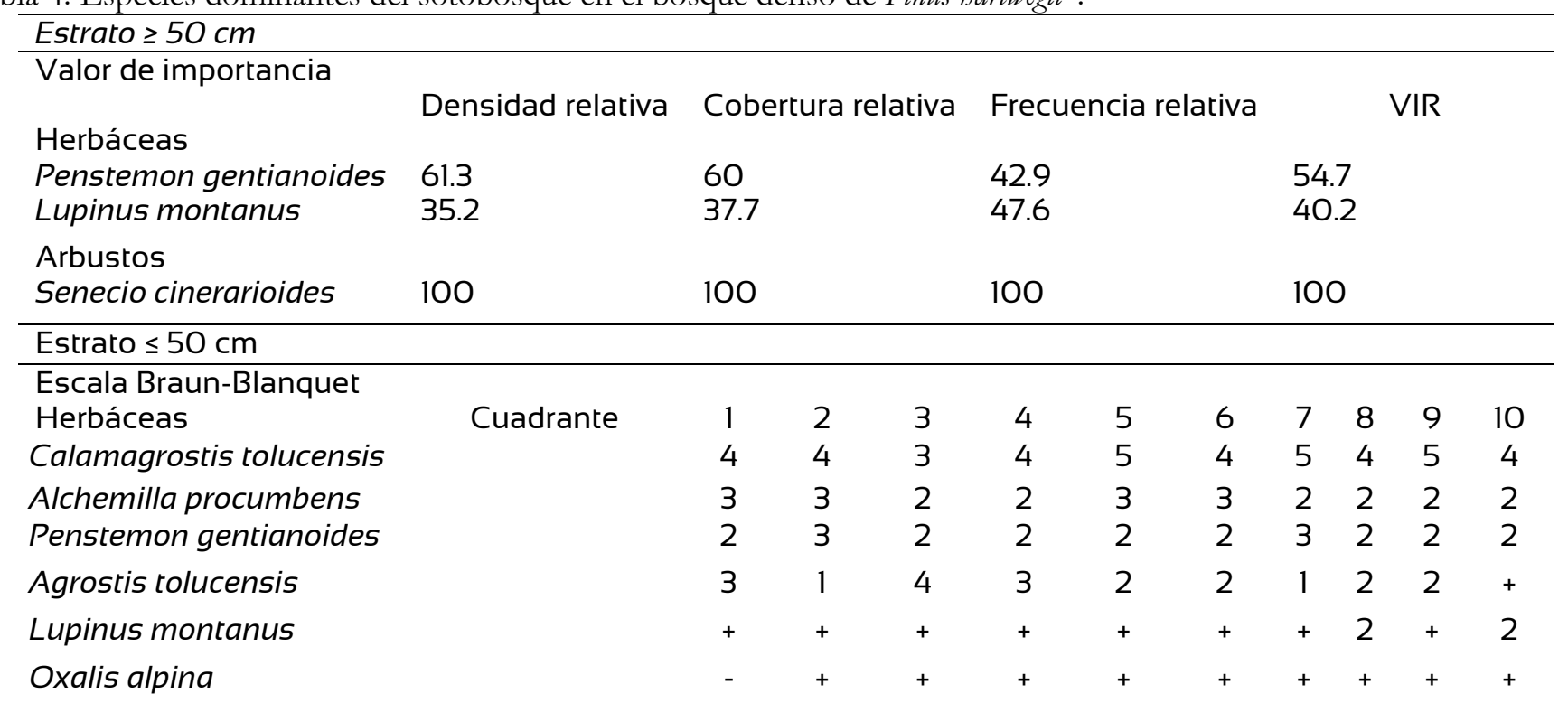

* Se indica el valor de importancia o la estimación de cobertura y abundancia.

"O" especies mayores a $50 \mathrm{~cm}$. Escala Braun-Blanquet: cobertura de la especie; (-) ausencia, (+) pocos individuos con baja cobertura, (1) <5\%, (2) 5 25\%, (3) 25 - 50\%, (4) 50 - 75\%, (5) 75\% - 100\%. Los resultados señalados en la tabla consideran a las especies dominantes, las especies restantes se omitieron por ser poco informativas. 


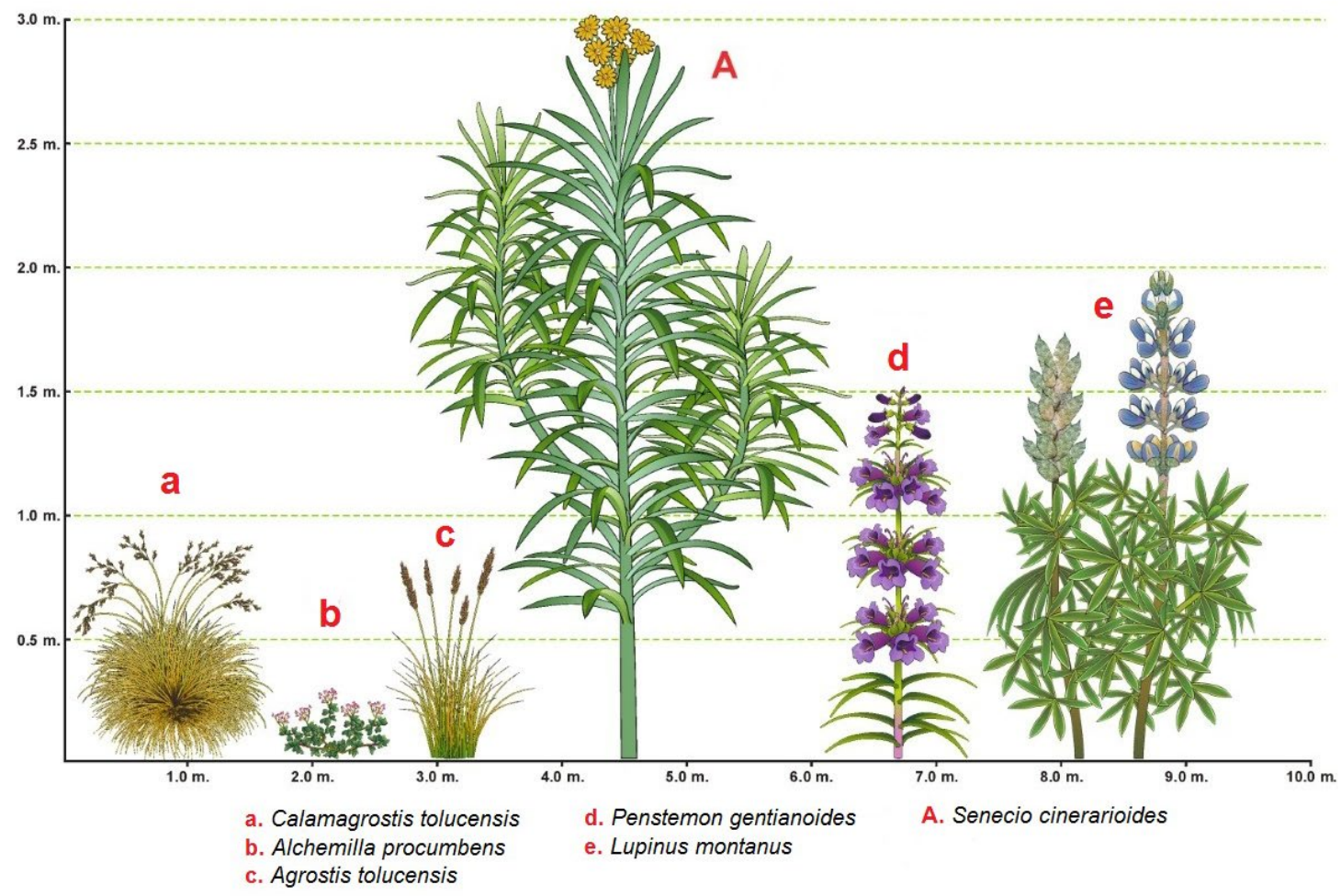

FIGURA 4. Estructura del sotobosque en el bosque denso de Pinus hartwegii, se presenta la altura y cobertura aproximada de las especies dominantes.

\section{DISCUSIÓN}

\section{Composición florística}

La vegetación del sotobosque en la comunidad de Abies religiosa posee una mayor riqueza de especies que la comunidad de Pinus hartwegii. Este dato concuerda con el registro de Villers, García y López (1998) en el Nevado de Toluca y con lo registrado por Vázquez (2014) en el Cofre de Perote, donde los bosques de Abies religiosa son más diversos y preceden en distribución altitudinal a los bosques de Pinus hartwegii.

La riqueza florística del bosque denso de Abies religiosa en el Nevado de Toluca es menor a la encontrada por ÁvilaAkerberg (2010) en la cuenca del río Magdalena (46 especies: 26 herbáceas, 15 arbustos y cinco árboles), pero mayor a la registrada por Vázquez (2014) en Veracruz con 27 especies (18 herbáceas, siete arbustos y dos árboles). Es posible que la riqueza de especies en el sotobosque de la especie esté determinada por múltiples factores, entre ellos la pendiente o la orientación (Gracia, Montané, Piqué y Retana, 2007; Huo, Feng y Su, 2014). Se trata de comunidades que se distribuyen principalmente en barrancas, cañadas o laderas, donde la pendiente llega a superar $40 \%$ y la humedad es elevada (Sánchez-González y López-Mata, 2003).

La riqueza de especies en el bosque denso de Pinus hartwegii en el Nevado de Toluca es menor al registro de Ávila-Akerberg (2010) en la cuenca del río Magdalena (47 especies) y a lo encontrado por Vázquez (2014) en el Cofre de Perote con 21 especies. En la cuenca del río Magdalena la flora cuenta con 42 herbáceas, cuatro arbustos y un árbol, mientras que en el Cofre de Perote existen 17 herbáceas, tres arbustos y un árbol. En los bosques de esta especie la riqueza florística suele estar condicionada a su distribución altitudinal. Hacia el límite superior de la vegetación arbórea, factores como la temperatura, la precipitación, la radiación solar y los nutrientes del suelo reducen el crecimiento de las plantas (Sharma, Suyal, Gairola y Ghildiyal, 2009). Además 
de estos factores, otros como el nivel de adaptación o tolerancia al ambiente de las especies, el tipo o estructura del dosel en el que se desarrollan y la disponibilidad de recursos, determinan la composición y estructura del sotobosque (North, Oakley, Fiegener, Gray y Barbour, 2005; Barbier, Gosselin y Balandier, 2008; Fuxai, Fousseni, Chungang, Huaijiang y Xiuhai, 2014).

En el sotobosque de ambas comunidades evaluadas la forma de vida que predomina es la herbácea, seguida de los arbustos y los árboles, resultado que avala el registro de Villaseñor y Ortiz (2014) para las plantas con flores del país. Así mismo la familia que reúne el mayor número de especies y endemismos es Asteraceae. En México, dicha familia representa la mayor riqueza florística y un elevado porcentaje de endemismos (Villaseñor, 2003; Villaseñor, Maeda, Colín y Ortiz, 2005). En el sotobosque de Abies religiosa, el género más representativo fue Roldana y en el sotobosque de Pinus hartwegii fue Senecio.

Además de la familia Asteraceae, en el sotobosque de Pinus hartwegii la familia Poaceae presenta un nivel de importancia significativo. En el ambiente la abundancia de gramíneas está asociada frecuentemente a la rápida adaptación o expansión que presentan y que les permite aprovechar los recursos del medio. Estas especies crecen en condiciones de deficiencia de drenaje, inundaciones periódicas o suelos con exceso de sales solubles, lo que las convierte en especies competidoras (Grime, 1989; Rzedowski, 2006). Es importante señalar que en ambos ecosistemas la mayor parte de las especies encontradas son de origen nativo, situación prevaleciente en gran parte del territorio nacional (Villaseñor, 2016).

Las especies tolerantes a la sombra (esciófitas) dominan la composición vegetal de ambas comunidades evaluadas, en el sotobosque de oyamel esta dominancia es justificable por la densidad de individuos que presenta el dosel $\left(637\right.$ ha $\left.^{-1}\right)$. Esta densidad es significativamente menor en el bosque de pino (336 ha-1) (Endara et al., 2012). En el bosque de oyamel, la estructura arbórea presenta copas simétricas o piramidales que se extienden e impiden el paso de luz hacia el sotobosque (Narave y Taylor, 1997), estas características explican el ambiente de sombra y humedad que se genera y que resulta ideal para las especies tolerantes. Por el contrario, en el bosque de Pinus hartwegii la estructura del dosel es irregular, con ramas cortas y extendidas (Narave y Taylor, 1997) que permiten que los rayos solares lleguen al suelo (Rzedowski, 2006). Esta condición promueve la dominancia en cobertura y abundancia de las especies heliófitas, que son, en su mayoría gramíneas y que presentan una gran afinidad a los altos niveles de luz (Grime, 1989). En las plantas, el grado de tolerancia a la sombra define su relación con otros organismos y con el medio. La sombra influye en los elementos bióticos y abióticos del bosque, determinando su estructura y diversidad (Blanco-García, Sáenz-Romero, Martorell, Alvarado-Sosa y LindigCisneros, 2011; Valladares, Laanisto, Niinemets y Zavala, 2016).

En relación a la regeneración, este presenta un patrón de crecimiento poblacional en forma de "J" invertida, donde la densidad se concentra en las clases diamétricas inferiores y disminuye paulatinamente. Esta situación ha sido observada para otros bosques de Pinus hartwegii (Endara et al., 2012; Murrieta-Hernández, Pineda-López, Noa-Carrazana, Mata-Rosas, Zulueta-Rodríguez y FloresEstévez, 2014) y Abies religiosa (Encina-Domínguez et al., 2008; Endara et al., 2012; Pineda-López, Ortega-Solis, Sánchez-Velásquez, Ortiz-Ceballos y Vázquez-Domínguez, 2013). La regeneración de una especie arbórea puede depender de múltiples factores, entre los que se encuentran el gremio ecológico (heliófita o esciófita), la tasa de crecimiento, mortalidad o depredación de las plántulas, la competencia inter e intraespecífica de los renuevos, así como variables ambientales, bióticas y antrópicas (Yamamoto, 2000; Villavicencio, Santiago, Godínez, Chávez y Toledo, 2012). La regeneración del bosque de pino depende especialmente de la luz (al ser una especie heliófita) y de la frecuencia de incendios superficiales, debido a que favorece la germinación de sus semillas (ÁvilaFlores, González-Tagle, Jiménez-Pérez, Aguirre-Calderón, Treviño-Garza y Vargas-Larreta, 2012; Robles-Gutiérrez, Velázquez-Martínez, Rodríguez-Trejo, Reyes-Hernández y Etchevers-Barra, 2016). En cambio, el oyamel puede regenerarse bajo su propio dosel o con una mayor 
abundancia en los claros del bosque (Lara-González, Sánchez-Velásquez y Corral-Aguirre, 2009).

\section{Estructura del sotobosque}

El patrón de dominancia encontrado en el sotobosque de Abies religiosa es distinto al reporte de Villers et al. (1998) para la condición de bosque denso en la misma área de estudio, donde Roldana barba-jobannis, Eupatorium mairetianum, Smilax sp. y Senecio callosus. fueron las especies dominantes. Dicho resultado concuerda con los reportes del sistema volcánico transmexicano (Sánchez-González, López-Mata y Granados-Sánchez, 2005), la Sierra Nevada (Sánchez-González y López-Mata, 2003) y el Cofre de Perote (Vázquez, 2014), donde algunas de las especies mencionadas constituyen un elemento importante de la flora.

En el sotobosque en el bosque denso de Pinus hartwegii estudiado la única especie arbustiva dominante es Senecio cinerarioides, mientras que en el estrato herbáceo predominan Alchemilla procumbens y Penstemon gentianoides. Esto concuerda con lo señalado por Villers et al. (1998), en la misma área de estudio, pero difiere de los hallazgos de Sánchez-González y López-Mata (2003), Santillana (2013) y Vázquez (2014) en distintas áreas, donde algunas de las especies dominantes son Acaena elongata, Baccharis conferta, Roldana angulifolia, Vaccinium geminiflorum y Bromus catharticus.

Los bosques analizados comparten, en el sotobosque, dos especies: Alchemilla procumbens y Senecio cinerarioides que están asociadas a perturbaciones. Además de estas especies existen Acaena elongata, Sigesbeckia jorullensis (en el oyamel) y Lupinus montanus (en el pino) que alcanzan una importancia significativa. En el ambiente, la presencia de algunas especies vegetales está relacionada con los eventos de perturbación o las prácticas de manejo que modifican la composición y la riqueza de la comunidad (North et al., 2005; Duguid, Frey, Ellum, Kelty y Ashton, 2013).

En el bosque de oyamel, las perturbaciones más frecuentes son la apertura de senderos y el pastoreo. En cambio, en el bosque de pino ocurren eventos de reforestación, ocoteo, construcción de zanjas de infiltración y pastoreo. De forma general, el pastoreo, la herbivoría o el fuego alteran el establecimiento, la sobrevivencia y el rendimiento de las plantas, sus efectos pueden ser positivos, negativos o generar una reacción directa e indirecta sobre la vegetación (Warner y Cushman, 2002; Husheer, Robertson, Coomes y Frampton, 2006; Mysterud, 2006). Las perturbaciones influyen también en el suelo, los nutrientes y las condiciones del agua (Pokhriyal, Chauhan y Todaria, 2012). Una perturbación en la composición vegetal, puede aumentar la riqueza de especies o reducirla, en ocasiones un nivel moderado de disturbio, genera una elevada riqueza (Royo, Collins, Adams, Kirschbaum y Carson, 2010). El daño de una perturbación depende de su extensión, intensidad o frecuencia (Augustine, Derner y. Milchunas, 2010), por ejemplo, en algunos casos la acción de los herbívoros aumenta la densidad o cobertura en las plantas y favorece la invasión de especies exóticas (Nai-Bregaglio, Pucheta y Cabido, 2002; Vázquez, 2002). En el bosque, el fuego es otro factor clave que modifica los depósitos de biomasa, altera el ciclo hidrológico y transforma la vegetación (Secretariat of Convention on Biological Diversity, 2001; Hoss, Lafon, Grissino-Mayer, Aldrich y DeWeese, 2008). Especialmente en el sotobosque evaluado de Pinus hartwegii, la presencia de Lupinus montanus indica quemas frecuentes y la abundancia de gramíneas favorece la propagación del fuego (Castañeda, Endara, Villers y Nava, 2015).

\section{CONCLUSIONES}

La presente investigación contribuye al conocimiento de la riqueza, dominancia y estructura de especies del sotobosque, en bosques densos de Abies religiosa y Pinus hartwegii del APFFNT. En las comunidades evaluadas, el sotobosque presenta la mayor diversidad florística y alberga una gran cantidad de especies nativas que le confieren una fisonomía estructural única a cada ecosistema.

En el sotobosque de Abies religiosa la riqueza de especies es mayor a la del sotobosque de Pinus hartwegii. En esta comunidad las plantas de la familia Asteraceae (Roldana angulifolia, Stevia monardifolia, Roldana platanifolia) son 
dominantes en la composición florística y algunas como Sigesbeckia jorullensis están asociadas a disturbio. En el sotobosque de Pinus hartwegii la familia Asteraceae fue dominante por número de especies, sin embargo, en cuanto a cobertura y abundancia las especies Calamagrostis tolucensis y Agrostis tolucensis, de la familia Poaceae son predominantes. En esta comunidad la especie arbustiva más importante, por su abundancia y dominancia fue Senecio cinerarioides. La regeneración arbórea en el sotobosque de las comunidades evaluadas es exclusiva de Abies religiosa y de Pinus hartwegii. En ambos bosques por orden de importancia en clase y tamaño se registró un mayor número de plántulas que de brinzales y latizales.

\section{RECONOCIMIENTOS}

Se agradece al Consejo Nacional de Ciencia y Tecnología por el financiamiento otorgado y al M. en C. Ricardo Vega Muñoz por el acceso a las instalaciones del Herbario Hortorio (CHAPA) del Colegio de Postgraduados; al M. en C. Lucio Lozada Pérez y al Biól. Jorge Fernando Rojas Gutiérrez por la determinación taxonómica de los ejemplares de la familia Asteraceae; a Raúl Rubio por el diseño de imágenes y a Leticia Bermúdez por la elaboración del mapa; y a la M. en C. A y R. N. Isabel Martínez de la Cruz por su asesoría en la determinación de algunos ejemplares.

\section{REFERENCIAS}

Angiosperm Phylogeny Group [APG IV]. (2016). An update of the Angiosperm Phylogeny Group classification for the orders and families of flowering plants: APG IV. Botanical Journal of the Linnean Society, 181, 1-20.

Antos, J. A. (2017). Understory plants in temperate forests. En J. N. Owens \& H. Gyde (Eds.), Forests and forest plants (pp. 118). Encyclopedia of life support systems (EOLSS). http://www.eolss.net/sample-chapters

Augustine, D. J., Derner, J. D., \&. Milchunas, D. G. (2010). Prescribed fire, grazing, and herbaceous plant production in shortgrass steppe. Rangeland Ecology \& Management 63(3), 317-323. doi: 10.2111/REM-D-09-00044.1

Augusto, L., Dupouey, J., \& Ranger, J. (2003). Effects of tree species on understory vegetation and environmental conditions in temperate forests. Annals of Forest Science, 60, 823-831. doi: 10.1051/forest:2003077

Ávila-Akerberg, V. (2010). Forest quality in the southwest of Mexico City. Assessment towards ecological restoration of ecosystem services. Culterra, 56, 1-2.

Ávila-Flores, D. Y., González-Tagle, M. A., Jiménez-Pérez, J., Aguirre-Calderón, O. A., Treviño-Garza, E. J., \& VargasLarreta, B. (2012). Estructura de rodales de Pinus hartwegii afectados por incendios utilizando parámetros de vecindad en la Sierra Madre Oriental, México. Tropical and Subtropical Agroecosystems, 15, 377-387.

Barbier, S., Gosselin, F., \& Balandier, P. (2008). Influence of tree species on understory vegetation diversity and mechanisms involved - a critical review for temperate and boreal forests. Forest Ecology and Management, 254(1), 1-15. doi: 10.1016/j.foreco.2007.09.038

Bartels, S. F., \& Chen, H. Y. H. (2010). Is understory plant species diversity driven by resource quantity or resource heterogeneity?. Ecology, 91 (7), 1931-1938.

Blanco-García, A., Sáenz-Romero, C., Martorell, C., AlvaradoSosa, P., \& Lindig-Cisneros, R. (2011). Nurse-plant and mulching effects on three conifer species in a Mexican temperate forest. Ecological Engineering, 37(6), 994-998. doi: 10.1016/j.ecoleng.2011.01.012

Braun-Blanquet, J. (1979). Fitosociologia. Bases para el estudio de las comunidades vegetales. Ediciones $\mathrm{H}$. Blume. Madrid, España.

Candan, F., Broquen, P., \& Pellegrini, V. (2006). Cambios en el sotobosque asociados al reemplazo de la vegetación natural por Pinus ponderosa Dougl. con diferentes manejos (SO de Neuquén, Argentina). Investigación Agraria: Sistemas y Recursos Forestales, 15(1), 50-65.

Castañeda, M. F., Endara, A. R., Villers, M. L., \& Nava, G. (2015). Evaluación forestal y de combustibles en bosques de Pinus hartwegii en el Estado de México según densidades de cobertura y vulnerabilidad a incendios. Madera y Bosques, 21(2), 45-58. doi: 10.21829/myb.2015.212444

Comisión Nacional de Áreas Naturales Protegidas 2013. Estudio Previo Justificativo para la Modificación de la Declaratoria del Parque Nacional Nevado de Toluca, ubicada en el Estado de México, México.

Cornejo-Tenorio, G., \& Ibarra-Manríquez, G. (2008). Flora ilustrada de la Reserva de la Biosfera Mariposa Monarca. México, D. F., México: Centro de Investigaciones en Ecosistemas, UNAM - Comisión Nacional para el Conocimiento y Uso de la Biodiversidad. 
Duguid, M. C., Frey, B. R., Ellum, D. S., Kelty M., \& Ashton, M. S. (2013). The influence of ground disturbance and gap position on understory plant diversity in upland forests of southern New England. Forest Ecology and Management, 303, 148-159. doi: 10.1016/j.foreco.2013.04.018

Encina-Domínguez, J. A., Encina-Domínguez, F. J. Mata-Rocha, E., \& Valdes-Reyna, J. (2008). Aspectos estructurales, composición florística y caracterización ecológica del bosque de oyamel de la Sierra de Zapalinamé, Coahuila, México. Boletín de la Sociedad Botánica de México, 83, 13-24.

Endara, A. R. (2010). Análisis de los procesos de recuperación en el bosque templado del Parque Nacional Nevado de Toluca. Tesis de doctorado, Universidad Autónoma del Estado de México, Toluca, México.

Endara, A. R., Franco, S., Nava, G., Valdez, J. I., \& Fredericksen, T. S. (2012). Effect of human disturbance on the structure and regeneration of forests in the Nevado de Toluca National Park, Mexico. Journal of Forestry Research, 23(1): 3944. doi: 10.1007/s11676-012-0226-8

Franco, S., Regil, H. H., González, C., \& Nava, G. (2006). Cambio de uso del suelo y vegetación en el Parque Nacional Nevado de Toluca, México, en el periodo 1972-2000. Investigaciones Geográficas, 61, 38-57.

Fuxai, X., Fousseni, F., Chungang, P., Huaijiang H., \& Xiuhai Z. (2014). Effect of overstory on the seasonal variability of understory herbs in primary broad-leaved Korean pine forest of Changbai Mountain. African Journal of Biotechnology, 13(11), 1223-1230. doi: 10.5897/AJB12.1013

Gobierno del Estado de México [GEM] (1999). Programa de manejo del Parque Nacional Nevado de Toluca, México. Biocenosis A.C.

Gilliam, F. S. (2007). The ecological significance of the herbaceous layer in temperate forest ecosystems. BioScience, 57(10), 845-858. doi: 10.1641/B571007

Givnish, T. J. (1988). Adaptation to sun and shade: a whole-plant perspective. Australian Journal of Plant Physiology, 15, 63-92.

Gracia, M., Montané, F., Piqué, J., \& Retana, J. (2007). Overstory structure and topographic gradients determining diversity and abundance of understory shrub species in temperate forests in central Pyrenees (NE Spain). Forest Ecology and Management, 242(2-3), 391-397. doi: 10.1016/j.foreco.2007.01.056

Grime, J. P. (1989). Estrategias de adaptación de las plantas y procesos que controlan la vegetación. México, D. F. México: Limusa.
Hart, S. A., \& Chen, H. Y. H. (2006). Understory vegetation dynamics of North American boreal forests. Critical Reviews in Plant Sciences, 25, 381-397. doi:10.1080/07352680600819286

Hoss, J. A., Lafon, C. W., Grissino-Mayer, H. D., Aldrich, S. R., \& DeWeese, G. G. (2008). Fire history of a temperate forest with an endemic fire-dependent herb. Physical Geography, 29(5), 424-441. doi: 10.2747/0272-3646.29.5.424

Huo, H., Feng, Q., \& Su, Y. (2014). The influences of canopy species and topographic variables on understory species diversity and composition in coniferous forests. The Scientific World Journal, 2014, 1-8. doi: $10.1155 / 2014 / 252489$

Husheer, S. W., Robertson, A. W., Coomes, D. A., \& Frampton, C. M. (2006). Herbivory and plant competition reduce mountain beech seedling growth and establishment in New Zealand. Plant Ecology, 183(2), 245-256. Doi: 10.1007/s11258-005-9036-9

Lara-González, R., Sánchez-Velásquez, L. R., \& Corral-Aguirre, J. (2009). Regeneration of Abies religiosa in canopy gaps versus understory, Cofre de Perote National Park, México. Agrociencia, 43(7), 739-747.

Mastretta, A., Cao, R., Arzeta, S. N., Quadri, P., Escalante, T., Arredondo, L., \& Piñero, D. (2014). ¿Será exitosa la estrategia del cambio de categoría para mantener la biodiversidad del Nevado de Toluca?. En L. E. Eguiarte (Eds.), Reflexiones sobre la ecología y la conservación en México (pp. 7-17). México: Instituto de Ecología-Universidad Nacional Autónoma de México.

Mostacedo, B. y Fredericksen, T. S. (2000). Manual de Métodos Básicos de Muestreo y Análisis en Ecología Vegetal. Proyecto de Manejo Forestal Sostenible (BOLFOR). Santa Cruz de la Sierra, Bolivia: Editora El País.

Murrieta-Hernández, D. M., Pineda-López, M. R., NoaCarrazana, J. C., Mata-Rosas, M., Zulueta-Rodríguez, R., \& Flores-Estévez, N. (2014). The structure of Pinus hartwegii at the Cofre de Perote, Veracruz, Mexico. Open Journal of Forestry 4(4): 291-301. doi: 10.4236/ojf.2014.44035

Mysterud, A. (2006). The concept of overgrazing and its role in management of large herbivores. Wildlife Biology, 12(2), 129$141 . \quad$ doi: 10.2981/09096396(2006)12[129:TCOOA] 2.0.CO;2

Nai-Bregaglio, M., Pucheta, E., \& Cabido, M. (2002). El efecto del pastoreo sobre la diversidad florística y estructural en pastizales de montaña del centro de Argentina. Revista 
Chilena de Historia Natural, 75, 613-623. Doi: 10.4067/S0716-078X2002000300012

Narave, H., \& Taylor, K. (1997). Pinaceae. Flora de Veracruz, 98.

North, M., Oakley, B., Fiegener, R., Gray A., \& Barbour, M. (2005). Influence of light and soil moisture on Sierran mixed-conifer understory communities. Plant Ecology, 177, 13-24. doi: 10.1007/s11258-005-2270-3

Pineda-López, M. R., Ortega-Solis, R., Sánchez-Velásquez, L. R., Ortiz-Ceballos, G., \& Vázquez-Domínguez, G. (2013). Estructura poblacional de Abies religiosa (Kunth) Schltdl. et Cham., en el Ejido el Conejo del Parque Nacional Cofre de Perote, Veracruz, México. Revista Chapingo Serie Ciencias Forestales y del Ambiente, 19(3), 375- 385. doi: 10.5154/r.rchscfa.2012.11.058

Pokhriyal, P., Chauhan, D. S., \& Todaria, N. P. (2012). Effect of altitude and disturbance on structure and species diversity of forest vegetation in a watershed of central Himalaya. Tropical Ecology, 53(3), 307-315.

Regil, H. H., Franco, S., Nava, G., \& Ordóñez, J. A. B. (2015). Evaluación de las técnicas para el estudio del cambio de ocupación de suelo y propuesta metodológica para solventar algunas de sus inconsistencias: el Parque Nacional Nevado de Toluca, México. GeoFocus, Revista Internacional de Ciencia y Tecnología de la Información Geográfica, 16, 61-86.

Robles-Gutiérrez, C. A., Velázquez-Martínez, A., RodríguezTrejo, D. A., Reyes-Hernández, V. J., \& Etchevers-Barra, J. D. (2016). Probability of mortality by fire damage of young Pinus hartwegii Lindl. trees in the Izta-Popo National Park. Revista Chapingo Serie Ciencias Forestales y del Ambiente, 22(2), 165-178. Doi: 10.5154/r.rchscfa.2015.08.034

Royo, A. A., \& Carson, W. P. (2006). On the formation of dense understory layers in forests worldwide: consequences and implications for forest dynamics, biodiversity, and succession. Canadian Journal of Forest Research, 36(6), 13451362. doi: 10.1139/X06-025

Royo, A. A., Collins, R., Adams, M. B., Kirschbaum C., \& Carson, W. P. (2010). Pervasive interactions between ungulate browsers and disturbance regimes promote temperate forest herbaceous diversity. Ecology, 91(1), 93-105. doi: $10.1890 / 08-1680.1$

Rzedowski, G. C. de, Rzedowski, J., \& colaboradores. (2005). Flora fanerogámica del Valle de México ( $2^{\mathrm{a}}$ ed., $1^{\mathrm{a}}$ reimp.). Pátzcuaro (Michoacán), México: Instituto de Ecología, A. C.-Comisión Nacional para el Conocimiento y Uso de la Biodiversidad.
Rzedowski, J. (2006). Vegetación de México (1 ${ }^{\mathrm{a}}$ ed. digital). México: Comisión Nacional para el Conocimiento y Uso de la Biodiversidad. Recuperado de http:/ / www.biodiversidad.gob.mx/publicaciones/librosD ig/pdf/VegetacionMxPort.pdf

Sánchez-González, A. y López-Mata, L. (2003). Clasificación y ordenación de la vegetación del norte de la Sierra Nevada, a lo largo de un gradiente altitudinal. Anales del Instituto de Biología, Serie Botánica, 74(1), 47-71.

Sánchez-González, A., López-Mata, L., \& Granados-Sánchez, D. (2005). Semejanza florística entre los bosques de Abies religiosa (H. B. K.) Cham. \& Schltdl. de la Faja Volcánica Transmexicana. Investigaciones Geográficas, 56, 62-76.

Santillana, M. E. (2013). Análisis del estado de conservación del bosque de Pinus hartwegii en una unidad de paisaje de la Cuenca del Río Magdalena, México, D.F. Tesis de Maestría, Universidad de Alcalá, España.

Secretariat of Convention on Biological Diversity (SCBD). 2001. Impacts of buman-caused fires on biodiversity and ecosystem functioning, and their causes in tropical, temperate and boreal forest biomes. CBD Technical series No. 5. Montreal, Canada.

Sharma, C. M., Suyal, S., Gairola, S., \& Ghildiyal, S. K. (2009). Species richness and diversity along an altitudinal gradient in moist temperate forest of Garhwal Himalaya. Journal of American Science, 5(5), 119-128. doi: $10.7537 /$ marsjas050509.15

The International Plant Names Index [IPNI]. 2017. The international plant names index. Recuperado de http://www.ipni.org/index.html.

Valdez, J. I. (2002). Aprovechamiento forestal de manglares en el Estado de Nayarit, costa Pacífica de México. Madera y Bosques, 8(Es1), 129-145.

Valladares, F., Laanisto, L., Niinemets, Ü., \& Zavala, M. A. (2016). Shedding light on shade: ecological perspectives of understorey plant life. Plant Ecology \& Diversity, 9(3), 237251. doi: $10.1080 / 17550874.2016 .1210262$

Vázquez, D. P. 2002. Multiple effects of introduced mammalian herbivores in a temperate forest. Biological Invasions, 4(1-2), 175-191. doi: 10.1023/A:102052292

Vázquez, J. (2014). Fenología reproductiva de las comunidades vegetales del Parque Nacional Cofre de Perote, Veracruz, México. Tesis de maestría, Instituto de Investigaciones Forestales, Universidad Veracruzana, Veracruz, México.

Villaseñor, J. L. (2003). Diversidad y distribución de las Magnoliophyta de México. Interciencia, 28(3), 160-167. 
Villaseñor, J. L. (2016). Checklist of the native vascular plants of Mexico. Revista Mexicana de Biodiversidad, 87(3), 559-902. doi: 10.1016/j.rmb.2016.06.017

Villaseñor, J. L., Maeda, P., Colín, J. J., \& Ortiz, E. (2005). Estimación de la riqueza de especies de Asteraceae mediante extrapolación a partir de datos de presenciaausencia. Boletín de la Sociedad Botánica de México, 76, 5-18. doi: $10.17129 /$ botsci.1700

Villaseñor, J. L., \& Ortiz E. (2014). Biodiversidad de las plantas con flores (División Magnoliophyta) en México. Revista Mexicana de Biodiversidad, 85, 134-142. Doi: $10.7550 / \mathrm{rmb} .31987$

Villavicencio, R., Santiago, A. L., Godínez, J. J., Chávez, J. M., \& Toledo, S. L. (2012). Efecto de la fragmentación sobre la regeneración natural en la Sierra de Quila, Jalisco. Revista Mexicana Ciencias Forestales, 3(11), 9-23.

Villers, L., García, L., \& López, J. (1998). Evaluación de los bosques templados en México: una aplicación en el Parque Nacional Nevado de Toluca. Investigaciones Geográficas, 36, 719.
Warner, P. J., \& Cushman, J. H. (2002). Influence of herbivores on a perennial plant: variation with life history stage and herbivore species. Oecologia, 132(1), 77-85. doi: 10.1007/s00442-002-0955-z

Yamamoto, S. (2000). Forest gap dynamics and tree regeneration. Journal of Forest Research, 5(4): 223-229. doi: 10.1007/BF02767114

Manuscrito recibido el 21 de febrero de 2017

Aceptado el 7 de marzo de 2018

Publicado el 13 de diciembre de 2018

Este documento se debe citar como:

Mejía C., A., Segio Franco-Maass' S., Endara A., A. R., \& Ávila A., V. (2018). Caracterización del sotobosque en bosques densos de pino y oyamel en el Nevado de Toluca, México. Madera y Bosques, 24(3), e2431656. doi: 10.21829/myb.2018.e2431656

Madera y Bosques por Instituto de Ecología, A.C. se distribuye bajo una Licencia Creative Commons Atribución-NoComercialCompartirlgual 4.0 Internacional. 